\title{
A Very Compact KHN Filter with Multidecade Tuning
}

\author{
Carlos Muñiz-Montero ${ }^{1}$, Alejandro Díaz-Sánchez ${ }^{1,2}$ \\ Electronics departments \\ 1. National Institute for Astrophysics Optics and Electronics \\ 2. Politechnical Institute of Puebla. \\ Puebla, MEXICO \\ cmuniz, adiazsan@inaoep.mx
}

\begin{abstract}
A very compact implementation of a multifunction Kerwin Huelsman Newcomb (KHN) filter that can be frequency tuned almost seven decades, from $0.2 \mathrm{~Hz}$ to $1 \mathrm{Mhz}$, is presented. Tuning is achieved by means of high-value, programmable active resistors biased using the quasi floating gate (QFG) technique and linearisated through capacitive gate voltage averaging. The circuit, realized in a $0.5 \mu \mathrm{m}$ standard CMOS technology using only four CMOS inverters, six small capacitors, six small resistors and two programmable active resistors, occupies a total area of $0.02 \mathrm{~mm}^{2}$, dissipates $3.45 \mathrm{~mW}$ and presents a dynamic range at $0.1 \%$ THD of 55.86.
\end{abstract}

\section{INTRODUCTION}

In low frequency applications, such as analog processing of biomedical signals, analog filters are used to reject unwanted signals, such that large DC offsets generated by sensors and flicker or out band noise. Additionally, the bandwidth of most of the biomedical signals is in the range of $0.1 \mathrm{~Hz}-10 \mathrm{kHz}$ and, because of the typical requirements of biomedical applications, programmability is mandatory [1]. Unfortunately, the design of programmable integrated filters with bandwidths below $1 \mathrm{kHz}$ is not trivial, especially if other design specifications such as low noise, low distortion, small area and reduced power consumption, must be satisfied. The main challenge comes from the lack of large time constants available in integrated circuits. To overcome this problem, impractical techniques have been used in the literature, among them: use of external capacitors (additional output pads), capacitance multiplication (coarse programmability that is only possible with discrete control) and techniques for current division and current cancellation (prone to present mismatch and large offset components) [2-3]. A more successful alternative that reaches until five decades of frequency tuning is the companding approach in log-domain processing, where the input is compressed, then nonlinearly processed, and finally expanded at the output, preserving dynamic range (at low supply voltages) and the overall linearity [4]. This approach exploits the exponential law of MOS transistors biased in weak inversion and frequently requires de use of Multiple Input Translinear Elements (MITE) realized with floating gate transistors (FGT) [4, 6].

Work supported by CONACyT, Mexico, under Grant I-37470-A

\author{
Ramón González-Carvajal \\ Department of Electronic Engineering \\ School of Engineering, University of Sevilla \\ Sevilla, SPAIN \\ carvajal@gte.esi.us.es
}

The main problem of all the previously mentioned approaches is the huge die area required, and the fact that only the log-domain filters reach multidecade tuning. This work proposes a very compact and extremely simple realization of a KHN filter (suitable for biomedical applications) with multidecade tuning that does not require of log-domain processing. The proposal is based on the use of very largevalued, programmable active resistors biased using the quasi floating gate (QFG) technique and linearisated through capacitive gate voltage averaging. A current mode version of this strategy was recently reported in [7]. The paper is organized as follows. Section II introduces the quasi ideal integrator used in the KHN filter synthesis of Section III. Simulation results are presented in Section IV. Finally, in Section V some conclusions are drawn.

\section{QUASI-IDEAL INTEGRATOR DESIGN}

Let's consider the quasi-ideal integrator shown in Fig. 1a. The CMOS inverter is considered for analysis purposes as an OPAMP of gain $A$ with the non-inverting input connected to $V_{D D} / 2 . R_{g}$ is a programmable active resistor controlled by the voltage $V_{c p}$. The corresponding transfer function is given by

$$
H(s)=\frac{V_{\text {out }}}{V_{\text {in }}}=-\frac{1}{s R_{g} C\left(1+\frac{1}{A}\right)+\frac{1}{A}} \approx-\frac{1}{s R_{g} C}, \quad A \rightarrow \infty
$$

The circuit implementations of the CMOS inverter and the active resistor $R_{g}$ are illustrated in figures $1 \mathrm{~b}$ and $1 \mathrm{c}$, respectively. The inverter is realized by using large transistors to reduce noise and mismatch contributions. Nevertheless, more elaborated amplifiers can be employed to obtain low voltage and/or low power versions of this integrator. $R_{g}$ is a three transistor version of the linearised quasi-floating gate resistor proposed in [8], but with the transistors $M_{p 2}$ biased in weak, moderate or strong inversion region, always in triode mode, by the voltage $V_{c p}$. Consequently, the effective resistance can be tuned from hundreds of kilo-ohms to the range 


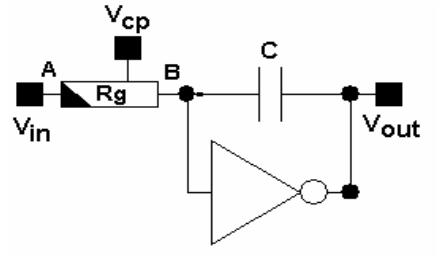

(a)

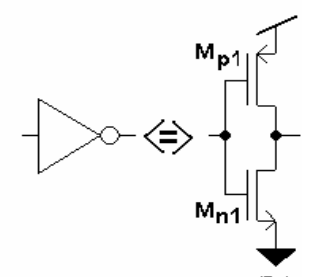

(b)

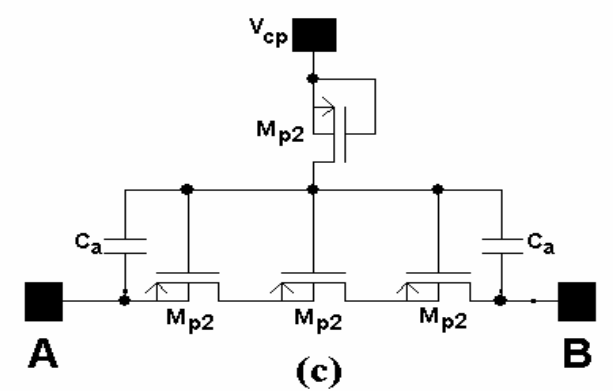

(c)

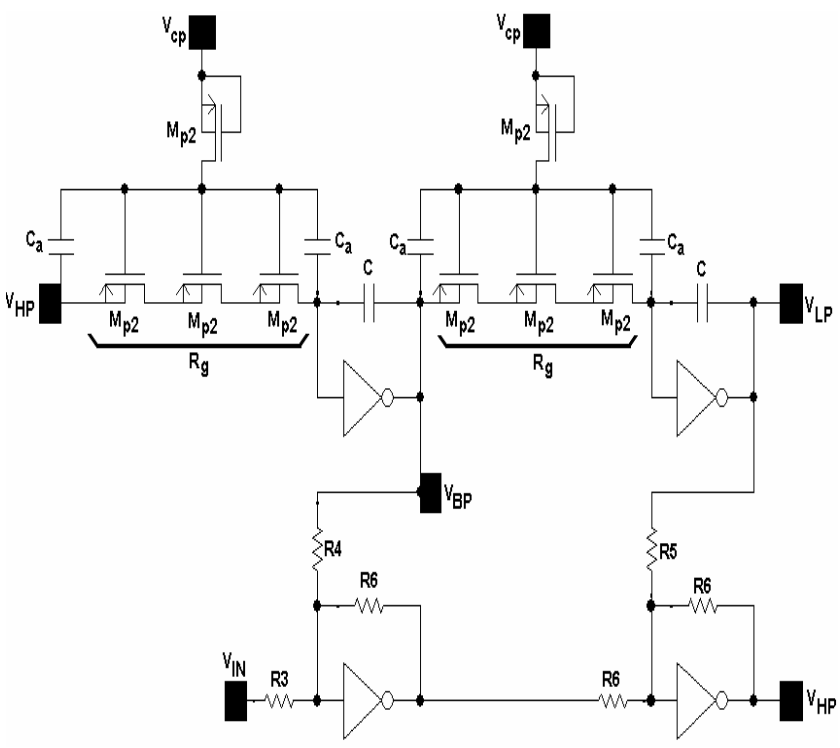

Figure 1. (a) Quasi-ideal integrator realized with a tunable active resistor. (b) CMOS inverter realization. (c) QFG programmable resistor linearised with the capacitive gate voltage averaging scheme proposed in [8]

of Gigaohms, while a capacitor of a few picofarads is enough to obtain cutoff frequencies in the order of hertz. Three transistors are necessary to avoid direct polarization of the parasitic active-substrate diodes due to signal fluctuations between the terminals of $R_{g}$, increasing the dynamic range [9, 10]. In addition, the weak-inversion operation of transistors $M_{p 2}$ forces $R_{g}<<R_{B}$ (where $R_{B}$ is the diffusion resistance), in order to avoid a parasitic resistive divider [11]. The substrate connection of the first transistor $M_{p 2}$ (marked with a black triangle in Figure 1a) should be connected to a drain instead of a gate to avoid unpredictable offsets due to substrate leakage currents [10].

In single-ended circuits with floating MOS resistors, linearisation can be performed by including in the gate voltage a component corresponding to the average of the voltages in the terminals of the resistor [8]. This averaging is achieved by the small capacitors $C_{a}$. Consequently, the gate voltage of transistors $M_{p 2}$ becomes $\left(V_{A}+V_{B}\right) / 2+V_{c p}$.

\section{KHN Filter With Multidecade Tuning}

The Kerwin Huelsman Newcomb (KHN) filter is a topology with extreme flexibility, good performance and low sensitivities [12]. A circuit approximation of this filter, using the quasi-ideal integrator described in the previous section, is illustrated in Figure 2. With a straightforward analysis, the high-pass, band-pass and low-pass responses becomes

$$
H_{H P}(s)=\frac{\frac{R_{6}}{R_{3}}\left(s+\frac{\psi}{A}\right)^{2}}{s^{2}+\psi\left(\frac{R_{6}}{R_{4}}+\frac{2}{A}\right) s+\psi^{2}\left(\frac{R_{6}}{R_{5}}+\frac{R_{6}}{R_{4} A}+\frac{1}{A^{2}}\right)}
$$

Figure 2. Tunable KHN filter realized with the quasi-ideal integrator of Figure 1a and the QFG programmable active resistor with capacitive gate voltage averaging linearisation of Figure 1c.

$$
\begin{gathered}
H_{B P}(s)=\frac{-\psi \frac{R_{6}}{R_{3}}\left(s+\frac{\psi}{A}\right)}{s^{2}+\psi\left(\frac{R_{6}}{R_{4}}+\frac{2}{A}\right) s+\psi^{2}\left(\frac{R_{6}}{R_{5}}+\frac{R_{6}}{R_{4} A}+\frac{1}{A^{2}}\right)} \\
H_{L P}(s)=\frac{\psi^{2} \frac{R_{6}}{R_{3}}}{s^{2}+\psi\left(\frac{R_{6}}{R_{4}}+\frac{2}{A}\right) s+\psi^{2}\left(\frac{R_{6}}{R_{5}}+\frac{R_{6}}{R_{4} A}+\frac{1}{A^{2}}\right)} \\
\psi=\frac{1}{R_{g} C}
\end{gathered}
$$

while the natural frequency $\omega_{n}$ and quality factor $Q$ are:

$$
\begin{gathered}
\omega_{n}=\psi \sqrt{\frac{R_{6}}{R_{5}}+\frac{R_{6}}{R_{4} A}+\frac{1}{A^{2}}} \\
Q=\frac{\sqrt{\frac{R_{6}}{R_{5}}+\frac{R_{6}}{R_{4} A}+\frac{1}{A^{2}}}}{\frac{R_{6}}{R_{4}}+\frac{2}{A}}
\end{gathered}
$$

From (6) and (7) is observed that the natural frequency can be tuned modifying the parameter $\psi=1 /\left(R_{G} C\right)$ through the voltage $V_{c p}$. Moreover, this tuning does not affect $\mathrm{Q}$. 


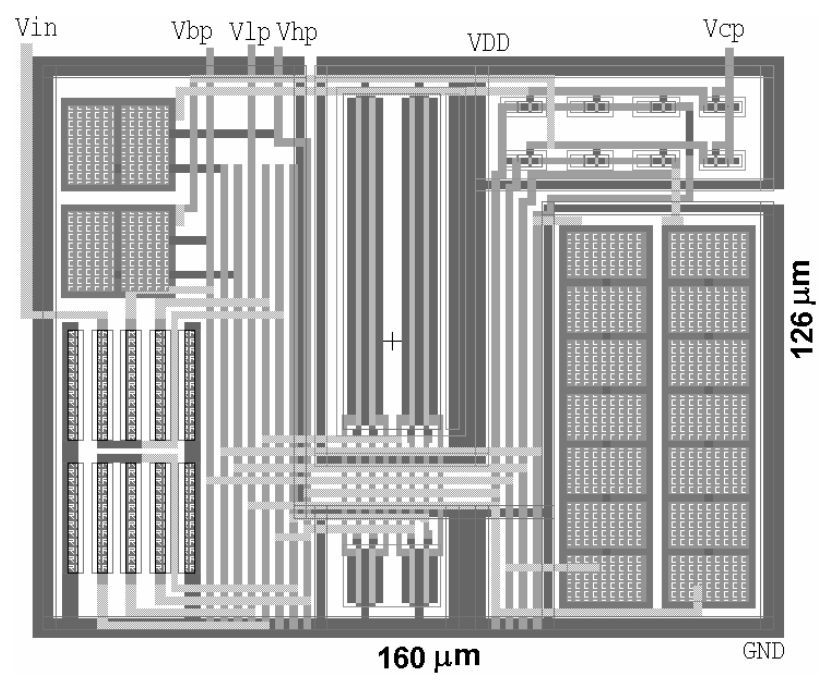

Figure 3. Layout of the programmable KHN filter of Figure 2 designed in a $0.5-\mu \mathrm{m}$ CMOS technology.

TABLE I. DESIGN DETAILS OF THE CIRCUIT OF FIGURE 2

\begin{tabular}{|c|c|}
\hline $\mathrm{M}_{\mathrm{n} 1}, \mathrm{M}_{\mathrm{p} 1}, \mathrm{M}_{\mathrm{p} 2}$ & $\mathrm{~W} / \mathrm{L}(\mu \mathrm{m} / \mu \mathrm{m})=$ \\
\hline $\mathrm{R}_{3}, \mathrm{R}_{4}, \mathrm{R}_{5}, \mathrm{R}_{6}$ & $10 \mathrm{~K} \Omega$ \\
\hline $\mathrm{C}, \mathrm{C}_{\mathrm{a}}, \mathrm{C}_{\mathrm{L}}$ & $1 \mathrm{pF}, 0.1 \mathrm{pF}, 1 \mathrm{pF}$ \\
\hline $\mathrm{V}_{\mathrm{DD}}, \mathrm{V}_{\mathrm{cp}}$, & $3 \mathrm{~V}, \mathrm{~V}_{\mathrm{cp}} \in(0 \mathrm{~V}, 1 \mathrm{~V})$ \\
\hline
\end{tabular}

\section{RESULTS}

The KHN filter of Figure 2 was designed using BSIM3.1 models for a $0.5 \mu \mathrm{m}$ CMOS AMI process $\left(\mathrm{V}_{\mathrm{THn}}=0.65 \mathrm{~V}\right.$, $\left|\mathrm{V}_{\text {THp }}\right|=0.95 \mathrm{~V}$ ). Figure 3 shows the layout of this filter using the design details summarized in Table I. All the capacitors are very small, consequently, the overall circuit occupies and area of only $0.02 \mathrm{~mm}^{2}$. The frequency responses for the lowpass, band-pass and high-pass signals are illustrated in Figure 4. Whit a sweep of $V_{c p}$ from 0 to $1 \mathrm{~V}$, the cutoff and center frequencies range goes from $0.2 \mathrm{~Hz}$ to $1 \mathrm{MHz}$, therefore featuring a tuning range of seven decades.

The simulated Total Harmonic Distortions (THD) of the low-pass and high-pass signals are shown in Figure 5 as a function of the amplitude of the input signal and the tuned frequency. The maximum levels of distortion appear with signals of frequency $0.1 \mathrm{~Hz}$ and amplitude above $0.4 \mathrm{~V}$. However, the THD remains in most of the cases below $1 \%$ ($40 \mathrm{~dB}$ ) with signals of amplitude below of $0.3 \mathrm{~V}$.

The input-referred noise spectral density of the low-pass signal is illustrated in Figure 6. The corresponding dynamic range, for a $100 \mathrm{~Hz}$-bandwidth (tuned frequency) and a signal of amplitude $100 \mathrm{mV}$ (corresponding to a THD of $0.1 \%$ ) and frequency $10 \mathrm{~Hz}$ is equal to $55.86 \mathrm{~dB}$.

In Table II some of the characteristics of tunable filters proposed in the literature are summarized and compared with the filter proposed in this work. As can be observed, the area

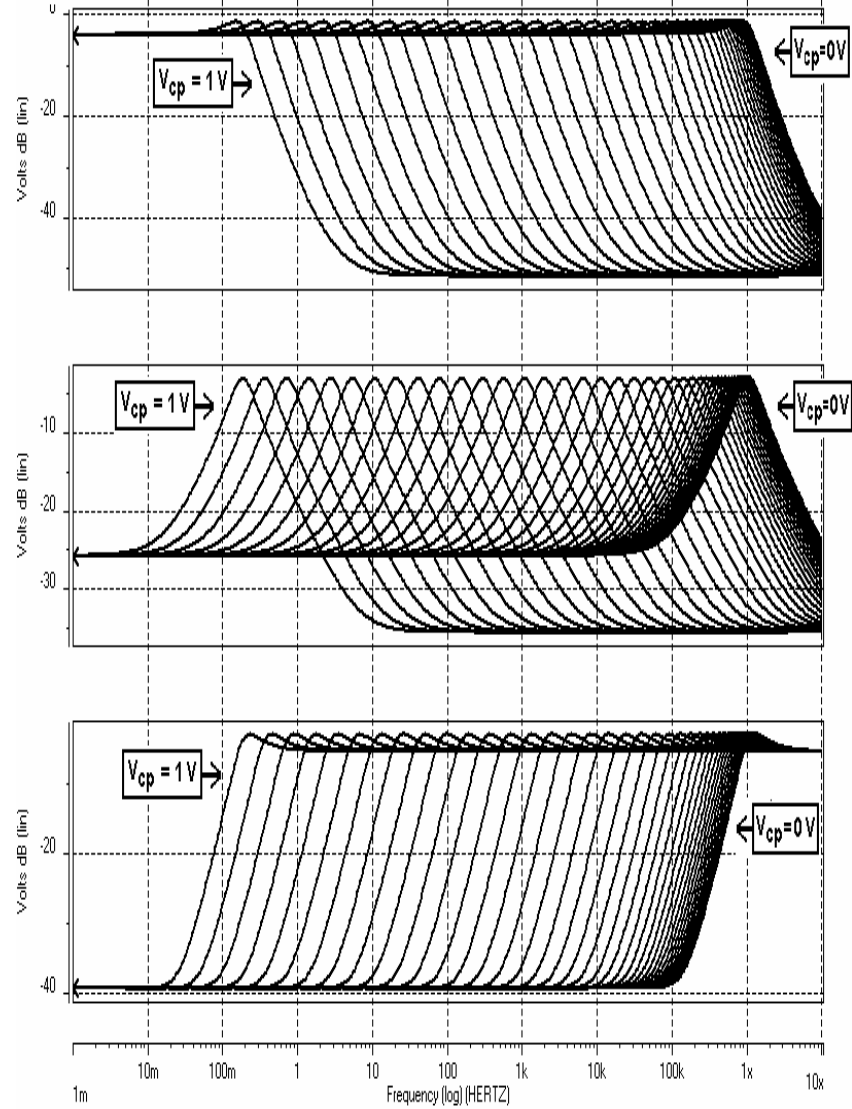

Figure 4. Low-pass, band-pass and high-pass responses of the KHN filter with a sweep of $V c p$ from $0 \mathrm{~V}$ to $1 \mathrm{~V}$ in increases of $25 \mathrm{mV}$.

of the proposed filter is only the $2 \%$ of the area reported in references [2] and [5], and the tunable range is two decades bigger than the range of the log-domain filter proposed in [4].

Finally, an average power consumption of $3.45 \mathrm{~mW}$ was obtained using the technique reported in [13]. Nevertheless, more elaborated amplifiers can be employed instead of the inverter to obtain a better power consumption.

\section{CONCLUSIONS}

A new strategy to design low-frequency, tunable integrated filters has been presented and verified by simulation. This technique uses large-valued active resistors to overcome the principal challenges of the previously reported strategies in the literature, allowing an extremely compact and easy circuit realization, with low hardware complexity, fully integrated capacitors and multidecade tuning capability without using the log-domain processing approach.

The strategy can be used in other filter topologies and scales with the CMOS processes. Also, the power consumption and voltage requirements can be easily reduced if more elaborated amplifiers are used instead of CMOS inverters. 


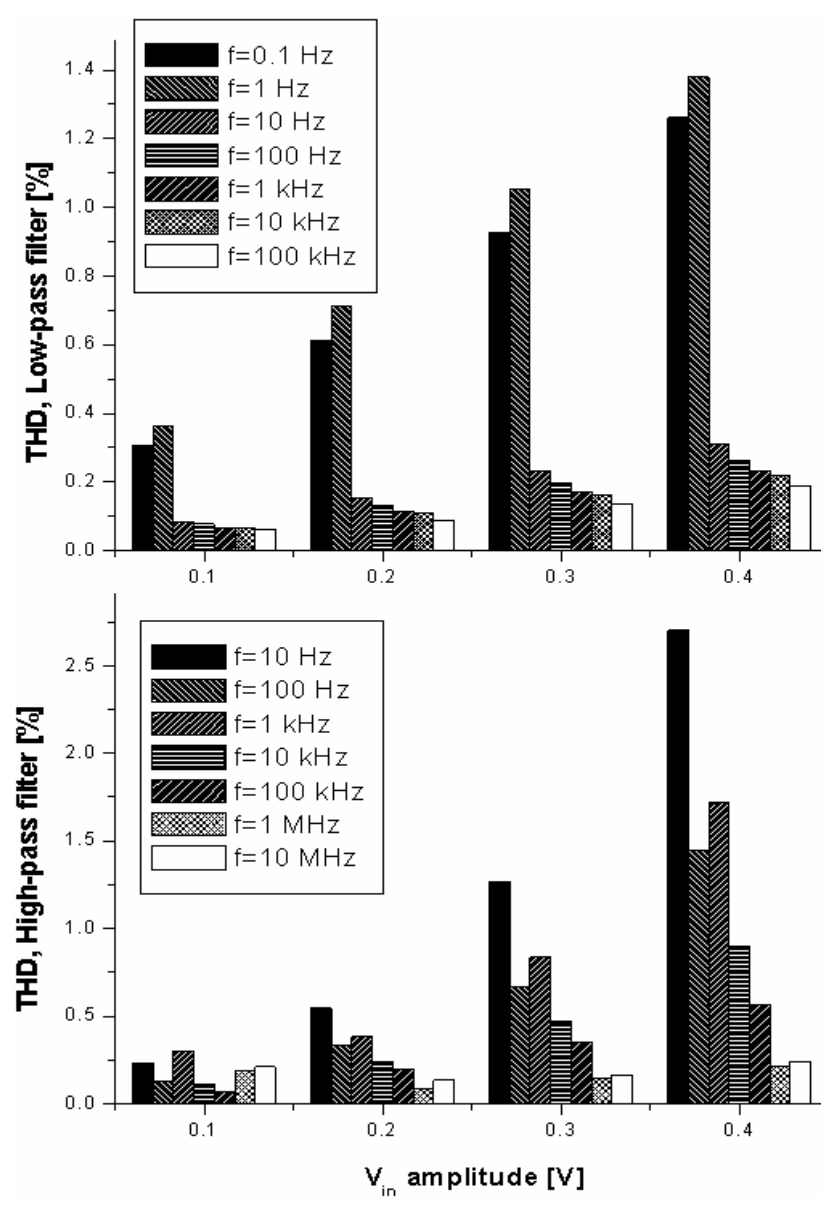

Figure 5. Simulated THD of the low-pass and high-pass signals as a function of the amplitude of the input signal and the tuned frequency. The frequency of the input is one decade below or above of the tuned frequency, for the case of the low-pass and high-pass filters, respectively.

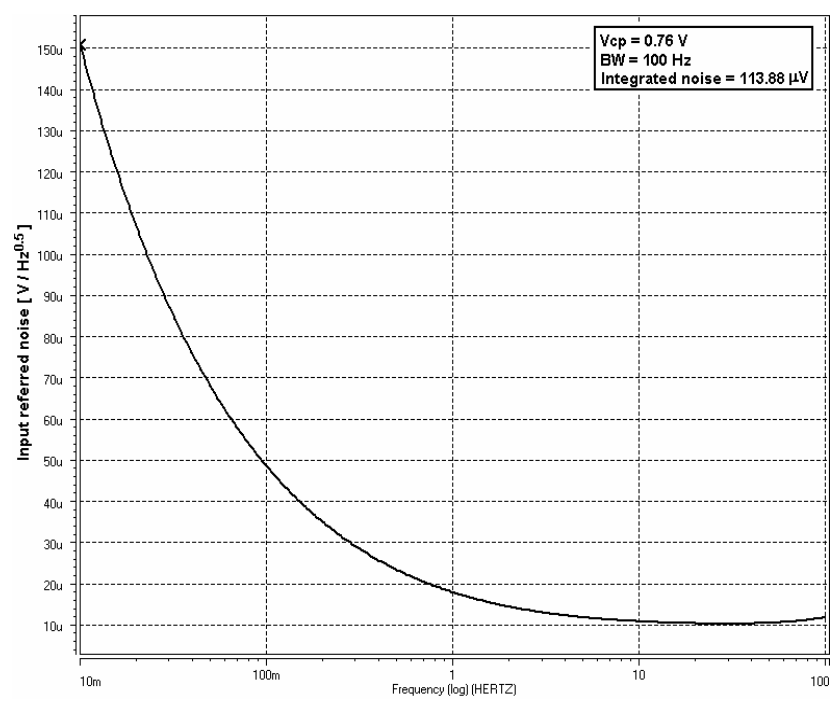

Figure 6. Input referred noise of the low-pass response tuned at $100 \mathrm{~Hz}$.
TABLE II. COMPARISON OF TUNABLE FILTERS IN THE LITERATURE

\begin{tabular}{|c|c|c|c|}
\hline $\begin{array}{c}\text { Reference } \\
\text { and } \\
\text { CMOS } \\
\text { process }\end{array}$ & Tunable range & Order & Die area \\
\hline $\begin{array}{c}{[2]} \\
(1.2 \mu \mathrm{m})\end{array}$ & $2 \mathrm{~Hz}$ & 6 & $1 \mathrm{~mm}^{2}$ \\
\hline $\begin{array}{c}{[4]} \\
(1.2 \mu \mathrm{m})\end{array}$ & $(1 \mathrm{~Hz}-100 \mathrm{kHz})$ & 2 & Not reported \\
\hline $\begin{array}{c}{[5]} \\
(0.8 \mu \mathrm{m})\end{array}$ & $(50 \mathrm{kHz}-2.1 \mathrm{MHz})$ & 3 & $1 \mathrm{~mm}^{2}$ \\
\hline $\begin{array}{c}{[6]} \\
(0.8 \mu \mathrm{m})\end{array}$ & $(25 \mathrm{~Hz}-35 \mathrm{kHz})$ & 1 & $0.1 \mathrm{~mm}^{2} *$ \\
\hline $\begin{array}{c}\text { This work } \\
(0.5 \mu \mathrm{m})\end{array}$ & $(0.2 \mathrm{~Hz}-1 \mathrm{MHz})$ & 2 & $0.02 \mathrm{~mm}^{2}$ \\
\hline
\end{tabular}

* Without consider two external capacitors of $150 \mathrm{pF}$

\section{REFERENCES}

[1] J. D. Bronzino, The Biomedical Engineering Handbook, CRC Press Inc. and IEEE Press, 1995.

[2] S. Solis-Bustos, J. Silva-Martínez, F. Malloberti and E. SánchezSinencio, "A 60 dB Dynamic Range Sixth Order 2.4 Hz Lowpass Filter for Medical Applications," IEEE Transactions on Circuits and Systems Part II, Vol. 47, No. 12, pp. 1391-1398, December 2000.

[3] J. Silva-Martínez and S. Solis-Bustos, "Designs considerations for high performance very low frequency filters," Proc. of the 50th International Symposium on Circuits and Systems, ISCAS 99, Vol II, pp. 648-651, Orlando, USA, June 1999.

[4] B. A. Minch, "Multiple-Input Translinear Element Log-Domain Filters," IEEE Transactions on Circuits and Systems-II: Analog and Digital Signal Processing, Vol. 48, No. 1, january 2001.

[5] J. A. De Lima and C. Dualibe, "A Linearly Tunable Low-Voltage CMOS Transconductor With Improved Common-Mode Stability and Its Application to gm-C Filters" IEEE Transactions on Circuits and Systems Part II, Vol. 48, No. 7, pp. 649-660, July 2001.

[6] A. J. López-Martín, C. A. De La Cruz-Blas, and A. Carlosena," 1.2 V 5 $-\mu W$ Class-AB CMOS Log-Domain Integrator With Multidecade Tuning" IEEE Transactions on Circuits and Systems Part II, Express Briefs, Vol. 52, No. 10, pp. 665-668, October 2005.

[7] C. Muñiz-Montero, R. González-Carvajal, A. Díaz-Sánchez and J. M. Rocha, "Low frequency, current mode programmable KHN filters using large-valued active resistors," In print, 58th International Symposium on Circuits and Systems, ISCAS 2007.

[8] J. Ramirez-Angulo, M. S. Sawant, R. G. Carvajal and A. López-Martín, "Linearization of MOS resistors using capacitive gate averaging," Electronics Letters, Vol. 41, No. 9, April 2005.

[9] C. Urquidi, J. Ramirez-Angulo, R. Gonzalez-Carvajal and A. Torralba, "A new family of low-voltage circuits based on quasi-floating gate transistors," in Proc. MWSCAS, Tulsa, OK, August 2002.

[10] I. Seo and R. M Fox, "Comparison of Quasi-/Pseudo-Floating Gate Techniques and Low-Voltage Applications, " in Analog Integrated Circuits and Signal Processing, V. 47, pp. 183-192. 2006.

[11] M. Bikumandla, J. Ramírez-Angulo, C. Urquidi, R. G. Carvajal and A. J. Lopez-Martin. "Biasing CMOS amplifiers using MOS transistors in subthreshold region," in IEICE Electronics Express, vol. 1 no. 12, September 25, 2004.

[12] L.P. Huelsman and P.E. Allen, Introduction to the theory and design of active filters, McGraw-Hill Book Co. USA, 1980.

[13] S.M. Kang, "Accurate Simulation of Power Dissipation in VLSI Circuits," IEEE Journal of Solid State Circuits, Vol. Sc-21, No. 5, pp. 889-891, October 1986. 